\title{
Ein Fall von Arrosion des Sinus mit Luftembolie und Pyämie mit tödlichem Ausgange.
}

Von

Dr. Schlegel, Braunschweig.

\begin{abstract}
Als Beitrag zur Klärung der in letzter Zeit mehrfach erörterten Frage von der Gefährlichkeit der Freilegung des gesunden Sinus scheint mir folgende Krankengeschichte von allgemeinerem Interesse zu sein.
\end{abstract}

H. Sch., 29 Jahre, aus gesunder Familie, hat im vierten Lebensjahre Scharlach mit rechtsseitiger Mittelohreiterung durchgemacht, welche in früheren Jahren häufig, im letzten Jahrzehnt seltener exzerbierte. Die vor mehreren Jahren von anderer Seite vorgeschlagene Operation wurde abgelehnt, da außer dem geringen Ausfluß keine Beschwerden bestanden. Seit einem halben Jahr stärkere Absonderung, seit mehreren Monaten viel rechtsseitigen, dumpfen Kopfschmerz, zuweilen leichtes Schwindelgefühl. Patient beabsichtigt in 4-5 Monaten als Farmer nach Südwest zu gehen und möchte vorher von seinem Leiden befreit sein.

10. Juli. Kaum mittelgroßer, schlanker Mann von grazilem Knochenbau, aber gut entwickelter Muskulatur, alle Organe gesund. L. O. normal.

R. O.: Trommelfell steht. Ubber dem kurzen Fortsatz großer, durch Granulationen verlegter Defekt der lateralen Attikwand, mit der Sonde Caries an der medialen und lateralen Wand nachweisbar.

Flüsterspr: : $1 / 4 \mathrm{~m}$, Weber $n$. R. Rinne -, Schwabach verlängert. C. bei mittelstark., $A_{1}$ bei leisem Anschlag.

13. Juli Operation: Nach dem ersten Meißelschlag Blutung aus dem vorgelagerten Sinus. Dieselbe steht nach kurzer Tamponade, und die Operation wird nun nach Stacke ausgeführt. Antrum, mittelgroß, tiefliegend und Aditus mit Granulationen ausgefullt, Hammer rudimentär, Amboß fehlt. Caries am Tegmen antri et tympani, welche in Markstückgröße entfernt werden. Dura leicht gerötet. Zur breiten Freilegung des Antrums muB auch der vorgelagerte Sulcus mit fortgenommen werden, so daß nach Vollendung der Operation der Sinus in seiner ganzen Breite und $1 \mathrm{~cm}$ Länge freiliegt. Am Schluß der Operation kann man deutlich die Vorlagerung des Sinus abschätzen. Der Abstand derselben von der hinteren Gehörgangswand beträgt nicht mehr wie $1-1 \frac{1}{2} \mathrm{~mm}$. Auffällig war auch die abnorme Dünne der Corticalis von höchstens $2 \mathrm{~mm}$, wodurch sich auch die Blutung bei dem ersten Meißelschlage erklärt. Die Stelle der Blutung am Sinus nicht nachweisbar, die Sinuswand sehr zart, so dab sich in Halbnarkose jeder Atemzug an dem wechselnden Füllungszustande des Sinu's erkennen läßt. 
Am 22., also am 9. Tage, wurde Patient aus der Klinik zur ambulanten Behandlung entlassen.

24. Juli. Verbandwechsel in der Sprechstunde. Mäßige, leicht fötide Sekretion. Während der Knochen überall mit frischen Granulationen bedeckt ist, zeigt die Sinuswand noch keine Neigung zum Granulieren. In der Mitte des freigelegten Sinus kleine linsengroße Partie leicht gelblich verfärbt. Lockere Tamponade mit in $\mathrm{H}_{2} \mathrm{O}_{2}$ getränkter Gaze.

26. Juli. Gestern nachmittag leichtes Frösteln und gestörtes Allgemeinbefinden. In der Nacht ist Patient aufgestanden, um Wasser zu trinken, stolpert uber einen Stuhl, hat nach dem Fall das Gefühl einer warmen Flüssigkeit im Ohr und denkt sofort an eine Blutung.

27. Juli 9 Thr morgens: Der Verband bis in die äuBeren Lagen mit Blut durchtränkt. Bei Herausnahme des über dem Simus liegenden Tampons entsteht ein schlürfendes Geräusch, Patient verfärbt sich, fallt vom Stuhle, so daß ich ihn gerade noch auffangen und auf den Boden legen kann. Dann stürat ein dicker Strahl venösen Blutes heraus. Durch festes Hineinpressen eines groBen Tupfers kommt die Blutung zum Stehen. Patient liegt während der wenigen Minuten, welche dieser Vorgang beansprucht, kollabient mit livider, leichenblasser Farbe da und kommt erst langsam wieder zu sich. Die Entfernung des Tampons aus dem Gehörgang gelingt, ohne daß es zur Blutung kommt. Nach dem Verbandwechsel leichter Schüttelfrost von 5 Minuten Daner. Wiederaufnahme in die Klinik.

27. Juli. Heute nacht subjektives Wohlbefinden. Temperatur $40,40,5,40,4$.

28. Juli. Gegen Morgen Schüttelfrost von knapp 10 Minuten Dauer. Tagsüber gutes Allgemeinbefinden, sehr guter Appetit. 'Temperatur $40,2,38,5,37,3$.

29. Juli. Bei Abnahme des Verbandes sofort wieder profuse Blutung. Durch Tamponade ron hinten stehend, sofort aber wieder einsetzend, als die Tamponarle vom Gehörgange aus entfernt wird. Der Versuch, den Sinus peripherwärts von der blutenden Stelle durch Einschieben eines Tampons zwischen Knochen und Sinus zu komprimieren, mißlingt, da die blutende Stelle bis an die Knochenwand reicht und beim geringsten Lüften des abschließenden Tampons sofort wieder starke Blutung einsetzt. Abends Schüttelfrost. Temperatur 40,2, $36,2,40,2$.

30. Juli. Nacht leidlich. Allgemeinbefinden wenig gestört, Appetit gut, klagt nur über Mattigkeit. Temperatur 40, 38,2, 39,8.

31. Juli. Operation: Nach Jugularisunterbindung Querschnitt nach hinten entsprechend dem Verlaufe des Sinus. Zirka $1 \mathrm{~cm}$ entfernt vom hinteren Rande der Operationshöle wird der Sinus in seiner ganzen Breite und 8-10 mam Länge freigelegt und durch Zwischenschieben eines dicken Tampons zwischen Knochen und Sinus fest komprimiert. Bei der nun folgenden Herausnahme der Tamponade aus der Ohroperationshöle wieder starke Blutung, anseheinend nur von unten kommend.

1. August. In der Nacht mehriach Erbrechen. Im Laufe des Tages Stimmung und Befinden gut, Nahrungsaufnahme sehr gut. Temperatur $37,8,39,2$.

2. August. Es gelingt, rom Gehörgang aus die Höhle zu übersehen. Pauke großenteils epidermisiert, im Aditus und medialen Teil des Antrum gesunde Granulationen. Bei dem Versuch, von hinten her durch Luiften des nach dem Bulbus zu liegenden Tampons die Sinuswand zu Gesicht zu bekommen, wieder starke Blutung, so daß der Tampon sofort wieder hineingedrückt werden muß. Temperatur $39,2,39,1,40$.

4. August. Frìh lang anhaltender Schüttelfrost. Netastase im 
rechten Hüftgelenk, in wenigen Tagen zu völliger Unbeweglichkeit führend, unter Ruhigstellung und Antiphlogese, aber nach acht Tagen abgeklungen. Temperatur 40,4, 38,6,40,6.

In der Nacht vom 4. zum 5. und am 5. August selbst noch je ein kürzerer Schüttelfrost.

6. August. Metastase im linken FuBgelenk, in zwei Tagen rorübergehend. Die Operationshöhle wird vom Gehörgang aus täglich verbunden. Die Epidermisierung macht gute Fortschritte. An der Jugulariswunde nichts Besonderes.

7. August. Der den Sinus zentralwärts abschließende Tampon wird entfernt ohne Blutung. Von unten her geringe fötide Sekretion, nach wenigen Tagen rexsiegend. An dem jetzt übersehbaren Sinus sieht man die laterale Wand in einer Länge von knapp $1 \mathrm{~cm}$ und dex ganzen Breite eingeschmolzen, so daß die mediale Wand in großer Ausdehnung freiliegt, die freien Ränder des Defektes wie ausgenagt.

9. August. Der den Sinus peripherwärts abschließende Tampon wird entfernt, Sinus verödet. Fs zeigt sich jetzt bei den Verbänden nicht ein Tropfen Blut mehr.

Vom 6. bis 19. August Continua meist zwischen 39-40. Das Allgemeinbefinden war trotz der hohen Temperatur in der ganzen Periode leidlich. Appetit wechselnd, meist aber zufriedenstellend. Sensorium immer frei, nie Kopfschmerzen. Auch der Kräftezustand war günsting bis zum 9. , wo starke, in den nächsten Tagen sich häufende blutige Durchfälle - am 10. und 11. zwischen 15 und 20 Stihle - mit ziemlich raschem Kräfteverfall einsetzten. Vom 15 . wieder Zunahme der Kräfte bei recht gutem Appetit.

Die Epidermisierung der Höhle hatte inzwischen einen normalen Verlauf genommen, Pauke, Aditus und der größere Teil des Antrum waren epidermisiert; die Jugulariswand bis auf einen oberflächlichen Streifen geschlossen.

19. August. Abends starker Schüttelfrost, am 20. früh Kollapstemperatur, dann täglich einen schweren Schüttelfrost. Unter rasch zunehmendem Kräfteverfall tritt am 23. früh der Exitus ein.

Sektion wurde leider nicht gestattet.

Der Fall erregt in mehrfacher Hinsicht unser Interesse. Zuerst durch die abnorme Lagerung des Sinus. Vorlagerungen des Sinus bekommt jeder Otochirurge mit größerem Material wohl häufiger zu Gesicht, ein derartig starkes Heranrücken des Sinus an die hintere Gehörgangswand dürfte aber doch zu den Seltenheiten gehören. In der mir zugängigen Literatur ist nur in zwei Fällen - Gruening und Muck - , ,Sinus der hinteren Wand unmittelbar anliegend" vermerkt, sonst findet sich meist angegeben: „Sinus vorgelagert oder stark vorgelagert". Mit diesen Angaben läßt sich aber wenig anfangen. Es würde sich empfehlen, in solchen Fällen das Verhältnis des Sinus zur hinteren Gehörgangswand durch Zahlen festzulegen.

Als eine unglückliche Folge der die breite Freilegung bedingenden Vorlagerung des Sinus ist die Arrosion zu betrachten. Daß Arrosionen des Sinus nicht so ganz selten sind, haben schon Eulenstein-Lebram durch Zusammenstellen von 20 Fällen, von denen aber nur 6 zur Operation kamen, bis zum Jahre 1905 nachgewiesen. In allen diesen Fällen handelte 
es sich aber entweder um Scharlachotitiden von besonderer Bösartigkeit oder um Fälle, bei welchen schon bei der Operation Veränderungen an der Sinuswand nachweisbar waren. In unserem Falle handelt es sich dagegen um einen gesunden Sinus, der durch die Einwirkung des von der Wundhöhle abgesonderten Sekretes arrodiert wird. Nun könnte man einwenden, daß die Arrosion durch die bei der Operation gesetzte Verletzung begünstigt worden sei. Gegen diese Auffassung spricht aber Versohiedenes. Die Verletzung bei der Operation kann nur an der Außenwand erfolgt sein, während die gelbliche Verfärbung sich zuerst an der medialen Wand zeigte. Ferner wäre es, wenn man die Stelle der Verletzung als Ausgangspunkt annehmen wollte, viel wahrscheinlicher zu einer Thrombose gekommen. Daß aber eine solche nicht bestand, geht aus dem ganzen Verlauf und späteren Befund hervor, wie auch aus dem Umstand, daß man bei den Verbänden den wechselnden Füllungszustand des Sinus bei jedem Atemzug beobachten konnte.

Grunert und Panse haben vor Jahren schon auf Grund je eines Falles, bei welchem ein bei der Operation völlig gesund befundener Sinus 16 resp. 14 Tage nach derselben wegen Thrombose ausgeräumt werden mußte, warnend darauf hingewiesen, den Sinus nicht mehr als zu vernachlässigende Größe zu betrachten, eine Warnung, die anscheinend wenig beachtet worden ist. In neuerer Zeit ist nun auch von Bondy im Gegensatz zu den Ausführungen von Marum durch eine Reihe einwandfreier Fälle der Nachweis erbracht, daß eine Thrombose als Folge der Freilegung eines gesunden Sinus eintreten kann. Diese Auffassung findet durch unseren Fall eine Stütze, bei welchem es zwar nicht zur Thrombose - die zweifellos günstiger gewesen wäre - , sondern gleich zu einer ausgedehnten Einschmelzung gekommen ist. Und eigentlich ist es wunderbar, daß ein derartiges unglückliches Vorkommnis nicht öfter beobachtet wird, da der Sinus bei seiner unbeständigen Lage doch gar nicht so selten auch unbeabsichtigt freigelegt wird und die Absonderung der Wundhöhle doch infektiös ist. Daß eine derartige Einwirkung des infektiösen Wundsekretes auf die Sinuswand im Verhältnis zur Thrombose die seltenere ist, geht auch aus den Haymannschen Versuchen hervor, bei dessen 13 Versuchstieren der Gruppe II - Aufbringen eines mit Bakterien getränkten infizierten Tampons auf den unverletzten Sinus - $12 \mathrm{mal}$ Thrombose, aber nur 3 mal eine Zerstörung der Sinusaußenwand zustande kam. 
Wahrscheinlich spielt die Stärke resp. Dicke der Sinuswand hier eine nicht unwesentliche Rolle. In unserem Falle war, wie gesagt, dieselbe so zart, daß man jeden einzelnen Atemzug am Sinus sich abspielen sah, wie ich es trotz einer großen Anzahl von Sinusfreilegungen in so ausgesprochenem Maße noch nie beobachtet hatte. Daß eine derartig zarte Wandung der Durchwanderung der Bakterien weniger Widerstand bietet, und andererseits längere Zeit zur Bildung eines schützenden Granulationswalles braucht, steht wohl außer Frage.

Das interessanteste, weil seltenste Phänomen in unserem Falle dürfte jedoch die Luftembolie sein: denn als eine solche muß der Zufall gedeutet werden, welcher sich beim Verbandwechsel am Tage nach der Blutung abspielte. Die hierbei auftretenden Erscheinungen - schlürfendes Geräusch, Stürzen des Patienten vom Stuble, Verfärbung und Kollaps, die nachfolgende Blutung - glichen den von Edgar Meier beschriebenen so, daß mein erster Gedanke war genau wie in dem Meierschen Fall. . Nach Tamponade auskultierte ich auch sofort das Herz und hörte zwar nur schwach, aber doch noch deutlich ein rasch verschwindendes gurgelndes Geräusch. Zweifellos ist aber nur wenig Luft aspiriert worden, wodurch es sich auch erklärt, daß der Vorgang nicht so schwere Erscheinungen hervorrief wie bei Meier und auch in erheblich kürzerer Zeit ablief, von dem Umstürzen des Patienten bis zum Aufstehen vergingen nur etwa 10-12 Minuten.

Bei einem unglücklich verlaufenen Fall quält man sich immer von neuem mit der Frage, hätte sich der traurige Ausgang vermeiden lassen, und was hätte denselben abzuwenden geschehen können?

Bezüglich einer ev. Schuldfrage kommen nun drei Momente in Betracht: die Freilegung des Sinus, die späte Unterbindung der Jugularis, die Unterlassung der Ausräumung des Bulbus.

Die Freilegung des Sinus kann in diesem Sinne nicht verwertet werden. Bei den abnormen anatomischen Verhältnissen lieB sich die breite Freilegung nicht umgehen, wenn man nicht auf eine gründliche Ausräumung des Krankheitsherdes verzichten wollte. Jedenfalls gibt aber der unglückliche Verlauf denen recht, welche gegen die unbeabsichtigte Aufdeckung des Sinus Front machen. Wenn derartige traurige Fälle auch sehr selten eintreten, so muB schon allein die Möglichkeit ihres Einsetzens von der Freilegung des Sinus - gesunden Sinus vorausgesetzt - abhalten. Weniger günstig liegen die Schuldverhältnisse hinsichtlich der Jugularisunterbindung. 
Auffällig war, daß der Sinus am 9. Tage nach der Operation noch nicht die geringste Neigung zum Granulieren zeigte. Zwei Tage später fiel mir unangenehm auf, daß ein minimaler Bezirk der Sinuswand gelblich verfärbt war, an die Möglichkeit derartiger schwerer Folgeerscheinungen dachte ich aber an diesem Tage noch nicht. Erst die Katastrophe am 26. machte mir die Größe der Gefahr klar. Ich zog schon jetzt die Jugularisunterbindung in Erwägung, nahm aber davon $\mathrm{Ab}$ stand in der Hoffnung, durch Tamponade die Blutung zum Stehen zu bringen und damit die Quelle der Infektion auszuschalten. Zu dieser Hoffnung verleitete mich eine Erfahrung, welche ich vor 3 Jahren erlebt hatte. Bei einer schweren Scharlachotitis trat 3 Wochen nach der Operation eine schwere einmalige Blutung mit zweimaligem Schüttelfrost binnen zwei Tagen auf, welche durch Tamponade zum Stillstand und ohne weitere Komplikation zur Ausheilung kam. Der Verlauf der nächsten Tage nahm mir aber diese Hoffnung, und am 31. führte ich die Jugularisunterbindung aus, leider zu spät. Hier, muß ich bekennen, liegt ein Verschulden meinerseits vor insofern, als ich die Unterbindung schon früher, nach dem zweiten oder spätestens dritten Schüttelfrost, hätte ausführen müssen. Der Verlauf des früheren Falles mag in etwas zu meiner Entlastung dienen, spricht mich aber nicht frei. Ob die frühere Unterbindung den traurigen Ausgang hätte abwenden können, ist ja freilich sehr fraglich; zweifellos wurden aber die Aussichten durch das lange Warten verschlechtert.

Über das Unterlassen einer ev. Bulbusoperation werden die Ansichten geteilt sein. Bei dem langen Intervall zwischen den Schüttelfrösten - vom 30. VII. bis 4. VIII. - erschien es mir sehr unwahrscheinlich, daß die neue Aussaat von dem Bulbus ausgegangen sein könnte.

Ich hielt mich auch auf Grund meiner sich allerdings nur auf zwei Fälle von Bulbusoperation erstreckenden ungünstigen Erfahrungen nicht für berechtigt, den Patienten noch einem so schweren Eingriff zu unterwerfen, dessen Nutzen mir gerade in diesem Falle mehr als zweifelhaft erschien.

\section{Literatur.}

1. Gruening, Bericht über die Verhandlungen der amerikanischen otologischen Gesellschaft. Z. f. O., Bd. 44.

2. Muck, Beitrag zur Kenntnis der gefährlichen Schläfenbeine. Z. f. O., Bd. 54 .

3. Meyer, Úber Luftembolie bei Sinusoperation. A. f. O., Bd. 49 . 
4. Lebram, Über Spontanblutung nach Arrosion des Sinus bei Scharlachotitis. Z. f. O., Bd. 50 .

5. Eulenstein, Über Blutungen infolge von Arrosion der Hirnblutleiter bei Eiterungen im Schläfenbein. Z, f. O., Bd. 43 .

6. Panse, Zwei Fälle von Sinusbeteiligung nach AufmeiBelung. A. f. O., Bd. 51.

7. Grunert, Jahresbericht der Kgl. Universitäts-Ohrenklinik $7 u$ Halle. A. f. O., Bd. 49.

8. Bondy, Zur Frage der Sinusthrombose und Freilegung des gesunden Sinus. A. f. O., Bd. 85 .

9. Marum, Jahresbericht über die Tätigkeit der UniversitätsPoliklinik \%u GieBen. A. f. O., Bd. 85.

10. Haymann, Sinusthrombose und otogene Pyämie im Lichte experimenteller Untersuchung. A. f. O., Bd. 83 . 